\title{
Relationship between Maternal Fetal Attachment and Self-Care Practices among Pregnant Women
}

\author{
Esraa Hamoda Mohamed Eltomy, Clinical Instructor \\ Obstetric and Gynecologic Nursing, Faculty of Nursing, Alexandria University \\ Rasha Mohamed Essa, Professor \\ Obstetric and Gynecologic Nursing, Faculty of Nursing, Damanhour University \\ Naglaa Fathy Fathalla Zaied, Lecturer \\ Obstetric and Gynecologic Nursing, Faculty of Nursing, Alexandria University
}

\begin{abstract}
Maternal-fetal attachment plays a key role in both the maternal and fetal health. Higher levels of maternal-fetal attachment can be considered as a great motivator for the woman to adopt good self-care practices which is the corner stones for safe passage through pregnancy. Objective: Identify the relationship between maternal-fetal attachment and self-care practices among pregnant women. Setting: Outpatient clinic of antenatal unit at El-Shatby Maternity University Hospital in Alexandria governorate. Subjects: A convenience sample of 375 pregnant women. Tools: Three tools were used for data collection: I. Basic Data Structured Interview Schedule, II. Maternal Fetal Attachment (MFA) Structured Interview Schedule, and III. Prenatal Self Care Practices Interview Schedule. Results: The present study findings showed a positive statistically significant correlation between pregnant women's overall maternal-fetal attachment level and their overall self-care practices. Conclusion: Maternal fetal attachment levels among the study subjects were significantly correlated with self care practices. Recommendations: Antenatal maternity nurses should consider the relationship between maternal fetal attachment and self care practices in their routine assessment and health education.
\end{abstract}

Keywords: Maternal fetal attachment; Self-care practices; Pregnant women.

\section{Introduction}

Pregnancy is a major life event for all women. It is a complex physiologic process that needs physical, emotional and psychological adaptation and adjustment to support the maternal-fetal unit. At no other time in women's life they experience such massive hormonal fluctuations as during pregnancy. Although it is described as a normal physiologic process, yet it is not without common discomforts and potentials for serious pregnancy related complications. Such complications usually augment the maternal morbidity and mortality rates ${ }^{(1)}$.

According to the World Health Organization (WHO 2017), the maternal mortality ratio (MMR) in Egypt was 37 maternal deaths $/ 100.000$ live births. In order to reduce the tocsin of maternal morbidity and mortality WHO had recommended several strategies one of them is the promotion of antenatal care ${ }^{(2)}$.

The goal of the ANC package is to prepare for birth as well as prevent, detect and manage pregnancy related health problems that affect mothers and their babies. The essential elements of this package are outlined in physical and psychological care. One of the most important psychological aspects of pregnancy is the development of maternal identity which is strengthened by a positive maternal-fetal attachment. This relationship starts before birth and lasts forever. After birth it is referred to as maternal - child bonding $^{(3)}$.

Maternal fetal attachment is defined as "a unique, gradual, reciprocal, emotional, warm, and close relationship between a pregnant woman and her fetus. It begins inutero and continues after birth". It is shaped 
by psychological, socio- demographic, reproductive factors as well as current pregnancy profile. As early as 1981, Cranley had developed the first maternal fetal attachment scale that measured attachment behaviors exhibited by pregnant women. It was classified into five behavioral domains: differentiation of self, maternal interaction with the fetus, attributing fetal characteristics, giving of self and role taking behaviors $^{(4)}$.

Self-care, in general, refers to a human regulatory function that is personally performed to maintain physical and psychological functioning. It supports personal development by managing internal and external factors that contribute to personal well-being. The benefits of self care practices include lower cost for the health care system, higher individual satisfaction and improve perception of one's health condition. According to Orem's work, Self care is performed to meet self care requisites. She further grouped these self care requisites into three groups: universal, developmental, and health deviation requisites. Where nurses' role mainly focuses on the universal one ${ }^{(6,7)}$.

In pregnancy, universal self care practices refer to all activities that the pregnant woman can follow in order to promote health and wellbeing of both herself as well as her fetus. Positive universal self care practices include: nutritious food, regular exercises, adequate rest and sleep, safety measures and hazard avoidance, hygienic care, seeking prenatal care and learning about pregnancy ${ }^{(8,9)}$.

It is very important for maternity nurses to understand the relationship between maternal fetal attachment and mothers' compliance with self-care practices. Such an understanding would pave the way for more sound ante-natal care and healthy pregnancy ${ }^{(10,11)}$.

\section{Aim of the Study}

The present study aimed to identify the relationship between maternal fetal attachment and self-care practices among pregnant women.

\section{Research Question}

Is there a relationship between maternal fetal attachment and self-care practices among pregnant women?

\section{Materials and Method}

\section{Materials}

Design: A descriptive correlational research design was utilized in this study.

Setting: This study was conducted at the outpatient clinic of antenatal unit at ElShatby Maternity University Hospital in Alexandria governorate.

Subjects: The study included 375 pregnant women who fulfil the following inclusion criteria:

- At 2nd and 3rd trimesters of pregnancy.

- Free from any chronic diseases.

- With normal course of pregnancy.

- Willing to participate in the study.

Epi info 7 program was used to estimate the sample size using the following parameters:

- Population size $=14000$ over 12 months.

- Expected frequency $50 \%$

- Acceptable error 5\%

- Confidence coefficient $95 \%$

- Minimal sample size 374.

Tools: Three tools were used for data collection:

\section{Tool I: Basic Data Structured Interview} Schedule

This tool was developed and used by the researcher to collect the basic data about the study subjects. It included three parts:

a) Socio-demographic characteristics.

b) Reproductive history.

c) Profile of current pregnancy. 


\section{Tool II: Maternal Fetal Attachment} (MFA) Structured Interview Schedule

This tool was adapted from two scales (cranley, 1981) and (Muller et al., 1993) and translated into Arabic language to measure the subjects' MFA level ${ }^{(12)}$. It entails 28 items. Subjects' response to each item varied among 3 point likert scale. Namely: never (1), sometimes (2), always (3). Each subject's total score ranged between 28 and 84. Each subject's MFA level was ranked as follows :

- Poorly attached $<47$.

- Moderately attached $47-<66$.

- Highly attached $\geq 66$.

\section{Tool III: Prenatal Self-Care Practices} Interview Schedule

This tool was originally developed by Lindgren (2001), and then revised (2005) to measure self care practices of pregnant women $^{(13)}$. It was adapted and translated to Arabic language by the researcher. It contained 26 items. Subjects' response to each item varied according to 3 point Likert scale. Namely: never (1), sometimes (2), and always (3). Each subject's total score ranged between 26-78. Accordingly each subject's self care practices level was ranked as follows :

- Poor self care practices $<43$.

- Fair self care practices $43-<60$.

- Good self care practices $\geq 60$.

\section{Method}

The study was conducted according to the following steps:

- Approval from ethical committee, Faculty of Nursing, Alexandria University was obtained.

- An official letter from the Faculty of Nursing, Alexandria University was directed to the responsible authorities of El- Shatby Maternity Hospital to take their permission for data collection.

- Tool (I) was developed by the researcher .
- Tool (II) and tool (III) were adapted and translated to Arabic language.

- Tools were tested for content validity by a jury of five experts in the field of obstetric and gynaecologic nursing.

- The tools' reliability was tested using internal consistency test (Cronbach $\alpha$ ) and the result was statistically accepted (tool II was 0.952 , tool III was 0.954 ).

- A pilot study was carried out on 38 women from the previously mentioned setting (excluded from the study subject) to ascertain the clarity, feasibility and applicability of the tools and to identify obstacles that might interfere with the process of data collection. After the pilot study, tools were revised and necessary modifications were done.

- Each woman was individually interviewed by the researcher in total privacy after explaining the purpose of the study and obtaining their consent. The duration of each interview ranged between 30-45 minutes. Three days per week (from 8:30 am to 1:30 pm) were specified for data collection over a period of four months, started from the beginning of July till the beginning of November. An average of 6 to 10 interviews was performed per day.

\section{Ethical considerations:}

For each recruited subject the following issues were considered:

- Securing the subject's informed written consent after explanation the aim of the study and the right to withdraw at any time.

- Keeping the subject's privacy.

- Assuring the subjects of their confidentiality. 


\section{Statistical Analysis}

The collected data was revised, categorized, coded and was fed to the computer and analyzed using Statistical Package for Social Science (SPSS) software version 20.0.

\section{Results}

Table (1) shows the socio demographic characteristics of the study subjects. More than one half $(56 \%)$ of them were in their twenties, $30.7 \%$ were in their thirties and only $13.3 \%$ were teenagers. About one-tenth $(9.6 \%)$ of them were illiterate or just read and write, compared to slightly less than two-fifths $(38.1 \%)$ holding secondary certificate. A close percentage $(37.9 \%)$ were university graduates. Approximately three-fifths $(60.8 \%)$ of them were housewives. Slightly more than one half $(54.4 \%)$ were dwelling urban areas. About fourfifth $(79.2 \%)$ of them perceived their family income as adequate.

Figure (1) exhibits the distribution of study subjects according to their maternal fetal attachment level. Slightly more than one half $(52.8 \%)$ of them were highly attached to their fetuses compared to $29.1 \%$ of them who were moderately attached. Only $18.1 \%$ of them were poorly attached to their fetuses.

Figure (2) exhibits the distribution of study subjects according to their self-care practices level. Slightly more than one half $(53.3 \%)$ of them were performing good self-care practices compared to about one-third (32.3\%) of them whose self-care practices were fair. Only $14.4 \%$ of them perform poor self-care practices during their pregnancies.

Table (2) shows the relationship between the maternal fetal attachment and self care practices levels. It demonstrates a positive statistically significant correlation between pregnant women's overall maternal fetal attachment level and their overall self care practices $(\mathrm{r}=0.874)$ with $(\mathrm{p}<0.001)$. That is to say an increase in maternal fetal attachment level is associated with an improvement in the women's self care practices.

According to table (3), a statistically significant positive correlation is observed between the overall level of maternal fetal attachment and three self care practices namely: a) nutrition b) safety measures and avoiding hazards c) seeking health care and information
( $\mathrm{p}<0.05)$. However, no statistically significant correlation is observed between the overall level of maternal fetal attachment and two self care practices namely: a) balance of rest and exercise b) personal hygiene $(\mathrm{p}>0.05)$.

\section{Discussion}

According to the results of the present study, slightly more than one half $(52.8 \%)$ of the study subjects were highly attached to their fetuses compared to $29.1 \%$ of them who were moderately attached. Similar findings were reported by Abasi et al. (2012) in their study titled "Assessment of effective factors of maternal fetal attachment in pregnant women" in $\operatorname{Iran}^{(14)}$. Their results showed that $55.4 \%$ of their study subjects were highly attached compared to $30.2 \%$ of them were moderately attached to their fetuses. This agreement between the results of the current study and the Iranian one may be attributed to the fact that both had used the same MFA scale and had collected their data from nearly equal numbers of study subjects.

On the other hand, this same result disagrees with the findings of Kaur \& Mamta (2017). They had conducted a comparative study to assess the maternal and paternal fetal attachment among the expectant mothers and fathers in Iran ${ }^{(15)}$. Their results showed that $90 \%$ of their female expectant mothers had strong maternal fetal attachment level. This disagreement here may be explained by the fact that the Iranian study here had used two different scales for measurement of maternal fetal attachment.

According to the findings of the present study, more than one half of the study subjects $(53.3 \%)$ were performing good self-care practices compared to $(32.3 \%, 14.4 \%)$ of them who were performing fair and poor self-care practices, respectively. This result is in harmony with the finding of a study conducted by Jihad \& Kadham in Iraq $(2016)^{(16)}$. It assessed self-care practices during prenatal period among mothers in Babylon city. They revealed that $50 \%$ of their study subjects were performing good overall selfcare practices compared to $(33.3 \%, 16.7 \%)$ of them were performing fair and poor self-care practices, respectively. This similarity between the two studies may be attributed to the cultural and sociodemographic homogeneity between the Egyptian and Iraqi populations.

On the other hand, this same result disagrees with the findings of Rizk et al. (2019) ${ }^{(17)}$. They had conducted a study in Yemen titled "Self-care 
practices utilized by Yemeni pregnant women in Hodeida city". They concluded that $40.6 \%$ of their study subjects were performing good universal self-care practices compared to $(58.2 \%, 1.2 \%)$ of them performed fair and poor universal self-care practices, respectively.

The discrepancy between the former study findings and the results of the present one, in this respect, could be explained by the difference in the data collection tools. They used pregnant women's self-care practices structured interview schedule that differs in items and scores from the currently used one.

In the light of the present study results, MFA level is positively correlated with the level of selfcare practices. That is to say, with increased levels of maternal fetal attachment, self care practices levels increase. These results are in harmony with the findings of Maddahi \& Dolatian (2016) ${ }^{(18)}$ who had conducted a study titled "Correlation of maternal fetal attachment and health practices during pregnancy with neonatal outcomes." in Iran.

This agreement between the previously mentioned studies and the current one could be explained by the relevant literature that suggests that higher levels of maternal fetal attachment may motivate the women to perform positive health behaviours during the pregnancy. Specifically, good nutrition, exercise, rest and sleep, safety practices and good hygiene,....etc. All these behaviours enhance the pregnant woman's health and reflect on her quality of life. Also, it plays a vital role in decreasing maternal mortality and morbidity.

In the light of the present study results, a statistically significant positive correlation was observed between the overall level of maternal fetal attachment and all of the following self care practices: (nutrition, safety measures, and seeking health care and information). These findings are nearly close to the findings of the previously mentioned study conducted in Iran by Maddahi \& Dolatian (2016) ${ }^{(18)}$. They had reported that safety practices and nutrition are statistically significantly positively correlated with maternal fetal attachment. Also, A British study titled "Maternalfetal attachment and engagement with antenatal advice" and conducted by Ross $(2012)^{(19)}$ had reported that adherence to health information and antenatal care in addition to hazards avoidance are statistically significantly correlated to maternal fetal attachment. Such an agreement, in this aspect, draws the attention to the fact that nutrition, safety measures, and seeking health care are very important practices that pregnant women should focus to keep their coming baby safe and in a good health.

On the other hand, the present study results reported no statistically significant correlation between the overall level of maternal fetal attachment and balance of rest and exercise in addition to personal hygiene. This same result nearly agrees with the findings of the previously mentioned Iranian study conducted by Maddahi \& Dolatian (2016) ${ }^{(18)}$. They reported that rest and exercise are not significantly correlated with maternal fetal attachment. Pregnant women's neglection of the importance of rest and exercise reflects her poor knowledge about its value for baby's health and wellbeing. These findings could be explained by the fact that hygiene may be a personal habit which is usually persistent and does not change during pregnancy. It means that the pregnant women may assume her usual hygienic measures either attached or not.

On the contrary, this result does not fit with the finding of Olander et al. (2018) ${ }^{(20)}$ in their study about health behaviours during pregnancy in The United Kingdom. They reported that exercise is significantly correlated with maternal fetal attachment. The disagreement between the British study and the current one may be explained by the difference in the study subjects' culture. Where exercise is highly regarded and is one aspect of a healthy lifestyle among the British culture.

\section{Conclusion}

Based on the findings of the present study, it could be concluded that a statistically significant positive correlation was observed between overall maternal fetal attachment level and self-care practices.

\section{Recommendations}

Based on the findings of the present study, the following recommendations are suggested:

- The issues of maternal fetal attachment and self care practices are recommended to be included within maternity nursing curricula at different nursing educational institutions.

- Assessment of maternal fetal attachment and self care levels are recommended to be included in the routine assessment of the antenatal maternity nurses.

- Antenatal health education should consider the positive relation between maternal fetal attachment and self-care practices which in turn improves the pregnancy outcome.

\section{For further researches:}

- Replication of the same study in different Egyptian cultures especially Upper Egypt or rural areas to compare and validate the present study findings.

- Replication of the same study on high risk pregnant women to include health deviation self care requisites in the study tool.

- Further studies to investigate the possible applicable strategies to strengthen the levels of maternal fetal attachment. 
Table (1): Number and percent distribution of the study subjects according to their sociodemographic characteristics

\begin{tabular}{|c|c|c|}
\hline Socio-demographic characteristics & $\begin{array}{c}\text { Number } \\
(375)\end{array}$ & Percent $(\%)$ \\
\hline \multirow[t]{2}{*}{$\begin{aligned} & \text { Age (years): } \\
&: \quad<20 \\
& \cdot 20-30 \\
& \cdot \geq 30\end{aligned}$} & $\begin{array}{c}50 \\
210 \\
115\end{array}$ & $\begin{array}{l}13.3 \\
56.0 \\
30.7\end{array}$ \\
\hline & \multicolumn{2}{|c|}{$\begin{array}{c}\text { Min. }- \text { Max }=16.0-40.0 \\
\text { Mean } \pm \text { SD }=26.25 \pm 5.70\end{array}$} \\
\hline $\begin{array}{l}\text { Level of education: } \\
\text { - Illiterate or read and write } \\
\text { - Primary level } \\
\text { - Secondary level } \\
\text { - } \quad \text { University or higher level } \\
\end{array}$ & $\begin{array}{c}36 \\
54 \\
143 \\
142 \\
\end{array}$ & $\begin{array}{c}9.6 \\
14.4 \\
38.1 \\
37.9 \\
\end{array}$ \\
\hline $\begin{aligned} & \text { Occupation: } \\
& \text { - } \text { Housewife } \\
& \text { - } \text { Worker } \\
& \text { - } \text { Employer } \\
&\end{aligned}$ & $\begin{array}{c}228 \\
66 \\
81 \\
\end{array}$ & $\begin{array}{l}60.8 \\
17.6 \\
21.6 \\
\end{array}$ \\
\hline \begin{aligned} \multicolumn{2}{l}{ Residence: } \\
- Urban \\
- Rural \end{aligned} & $\begin{array}{l}204 \\
171\end{array}$ & $\begin{array}{l}54.4 \\
45.6\end{array}$ \\
\hline $\begin{aligned} & \text { Perceived income: } \\
& \text { - } \text { Adequate } \\
& \text { - } \text { Not adequate } \\
&\end{aligned}$ & $\begin{array}{c}297 \\
78\end{array}$ & $\begin{array}{l}79.2 \\
20.8 \\
\end{array}$ \\
\hline
\end{tabular}

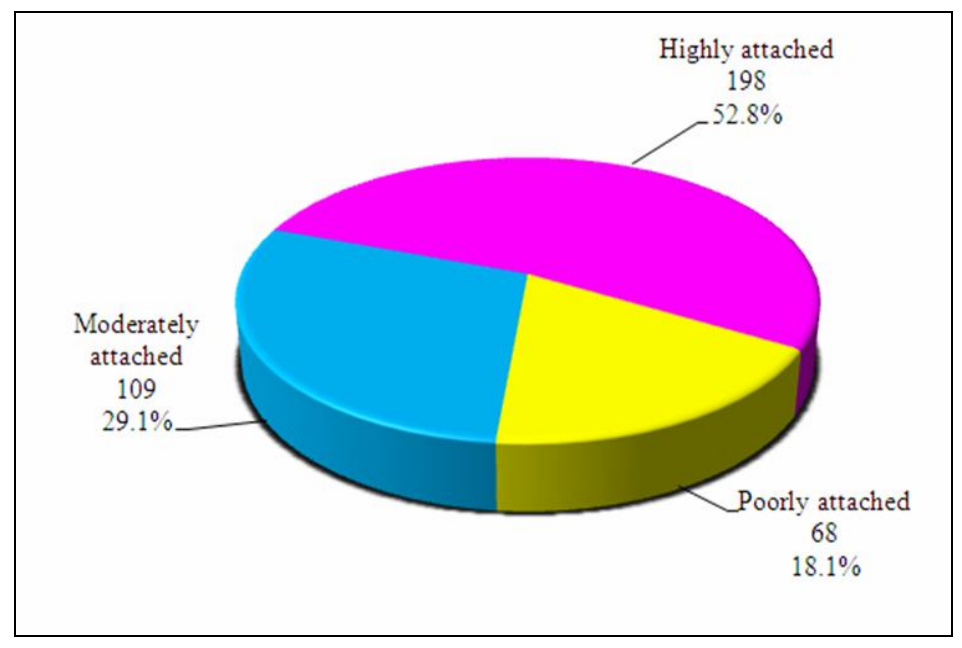

Figure (1): Percent distribution of the study subjects according to their maternal-fetal attachment (MFA) level 


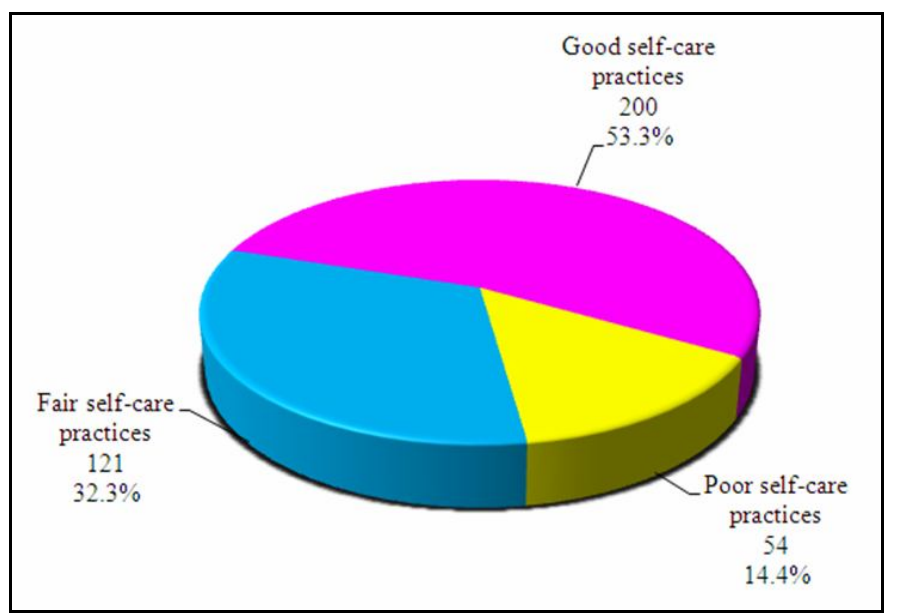

Figure (2): Percent distribution of the study subjects according to their overall prenatal self-care practices level

Table (2): Correlation between overall maternal fetal attachment and overall prenatal selfcare practices levels

\begin{tabular}{|c|c|c|c|c|c|c|c|}
\hline \multirow{3}{*}{$\begin{array}{l}\text { Prenatal self-care practices } \\
\text { levels }\end{array}$} & \multicolumn{6}{|c|}{ Maternal fetal attachment (MFA) levels } & \multirow{3}{*}{$\mathbf{p}$} \\
\hline & \multicolumn{2}{|c|}{$\begin{array}{c}\text { Highly attached } \\
(\mathbf{n}=198)\end{array}$} & \multicolumn{2}{|c|}{$\begin{array}{c}\text { Moderately } \\
\text { attached } \\
(\mathrm{n}=109) \\
\end{array}$} & \multicolumn{2}{|c|}{$\begin{array}{l}\text { Poorly attached } \\
(n=68)\end{array}$} & \\
\hline & No. & $\%$ & No. & $\%$ & No. & $\%$ & \\
\hline Good self-care practices & 189 & 95.5 & 5 & 4.6 & 6 & 8.8 & \multirow{3}{*}{$<0.001^{*}$} \\
\hline Fair self-care practices & 7 & 3.5 & 100 & 91.7 & 14 & 20.6 & \\
\hline Poor self-care practices & 2 & 1.0 & 4 & 3.7 & 48 & 70.6 & \\
\hline $\mathbf{r}_{\mathrm{s}}(\mathbf{p})$ & & & 0.874 & $0.001^{*}$ & & & \\
\hline
\end{tabular}

*: Statistically significant at $p \leq 0.05$

Table (3): Correlation between overall maternal fetal attachment and prenatal self-care practices' subscales

\begin{tabular}{|c|c|c|c|c|c|}
\hline \multirow{3}{*}{\begin{tabular}{|l} 
\% Score \\
Prenatal self-care practices
\end{tabular}} & \multicolumn{3}{|c|}{ Overall Maternal fetal attachment } & \multirow{3}{*}{$\mathbf{F}$} & \multirow{3}{*}{$\mathbf{p}$} \\
\hline & $\begin{array}{c}\text { High } \\
(\mathrm{n}=198)\end{array}$ & $\begin{array}{c}\text { Moderate } \\
(\mathrm{n}=109)\end{array}$ & $\begin{array}{c}\text { Low } \\
(n=68)\end{array}$ & & \\
\hline & Mean \pm SD. & Mean \pm SD. & Mean \pm SD. & & \\
\hline Nutrition & $66.79 \pm 20.37$ & $65.29 \pm 17.51$ & $59.07 \pm 25.30$ & $3.581^{*}$ & $0.029^{*}$ \\
\hline $\begin{array}{l}\text { Balance between rest and } \\
\text { exercise }\end{array}$ & $58.84 \pm 31.50$ & $57.57 \pm 25.57$ & $49.45 \pm 36.04$ & 2.403 & 0.092 \\
\hline $\begin{array}{l}\text { Safety measures and } \\
\text { avoiding hazards }\end{array}$ & $94.19 \pm 13.29$ & $60.73 \pm 21.55$ & $30.74 \pm 27.55$ & $310.567^{*}$ & $<0.001^{*}$ \\
\hline $\begin{array}{l}\text { Seeking health care and } \\
\text { information }\end{array}$ & $77.96 \pm 16.69$ & $45.54 \pm 17.41$ & $25.42 \pm 19.95$ & $272.746^{*}$ & $<0.001^{*}$ \\
\hline Personal hygiene & $75.19 \pm 27.95$ & $69.95 \pm 19.85$ & $67.65 \pm 26.38$ & 2.850 & 0.059 \\
\hline
\end{tabular}

F: F for ANOVA test

*: Statistically significant at $p \leq 0.05$ 


\section{References}

1. SOMA-PILLAY, Priya. Physiological changes in pregnancy. Cardiovascular Journal of Africa 2016; 27(2): 89.

2. WORLD HEALTH ORGANIZATION. Trends in maternal mortality 2000 to 2017: estimates by WHO, UNICEF, UNFPA, World Bank Group and the United Nations Population Division 2019.

3. Ngxongo TS. Basic Antenatal Care Approach to Antenatal Care Service Provision. In Selected Topics in Midwifery Care 2018 Nov 5. IntechOpen.

4. Welch L, Miller L. Emotional and educational components of pregnancy. Glob. libr. Women's Med., (ISSN: 1756-2228). 2008.

5. Homer CS, Oats J, Middleton P, Ramson J, Diplock S. Updated clinical practice guidelines on pregnancy care. Medical Journal of Australia 2018; 2009(9): 409-12.

6. Salehi K, Kohan S. Maternal-fetal attachment: What we know and what we need to know. International Journal of Pregnancy \& Child Birth 2017; 2(5): 00038.

7. Ghodrati F, Akbarzadeh M. A review of the importance of maternal-fetal attachment according to the Islamic recommendations. Journal of Midwifery and Reproductive Health 2018; 6(1): 1193-200.

8. BRESSI, Sara K.; VADEN, Elizabeth R. Reconsidering self care. Clinical Social Work Journal 2017; 45(1): 33-38.

9. Younas A. A foundational analysis of Dorothea Orem's self-care theory and evaluation of its significance for nursing practice and research. Creative nursing 2017; 23.1: 13-23.

10. Zhianian A, et al. Improving self-care behaviors in pregnant women in Zahedan: Applying selfefficacy theory. Caspian Journal of Health Research 2015; 1(1): 18-26.

11. Mannava P, Durrant K, Fisher J, Chersich M, Luchters S. Attitudes and behaviors of maternal health care providers in interactions with clients: a systematic review. Globalization and health $2015 ; 11(1): 36$.
12. Hassan NM, Hassan FM. Predictors of maternal fetal attachment among pregnant women. IOSR J Nurs Heal Sci [Internet] 2017; 6(1): 95-106.

13. Gomora A, Mukona D, Zvinavashe M, Dhaka A. The relationship between prenatal self care and adverse birth outcomes in young women aged 16 to 24 years. Journal of Nursing and Health Science 2015; 4(1):72-80.

14. Abasi E, Tahmasebi H, Zafari M, Takami GN. Assessment on effective factors of maternalfetal attachment in pregnant women. Life Science Journal 2012; 9(1): 68-75.

15. Kaur S, Sagar MN. Comparative study to assess the maternal and paternal fetal attachment among the expectant mothers and fathers. Int J Reprod Contrcept Obstet Gynecol 2017; 6(7): 3134-7.

16. Jihad SK, Kadham NM. Assessment of SelfCare Practices during Prenatal Period among Mothers in Babylon City. kufa Journal for Nursing sciences. 2016; 6(1): 209-15.

17. Rizk SA, Ghaly AS, Youssef HI. Self-Care Practices Utilized By Yemeni Pregnant Women in Hodeida City. Journal of Nursing and Health Science2019; 4(2): 20-32.

18. Maddahi MS, Dolatian M. Correlation of maternal-fetal attachment and health practices during pregnancy with neonatal outcomes. Electronic physician 2016; 8(7): 2639.

19. Ross E. Maternal-fetal attachment and engagement with antenatal advice. British Journal of Midwifery 2012; 20(8): 566-75.

20. Olander EK, Smith DM, Darwin Z. Health behavior and pregnancy: a time for change 2018; 6(2): 23 . 